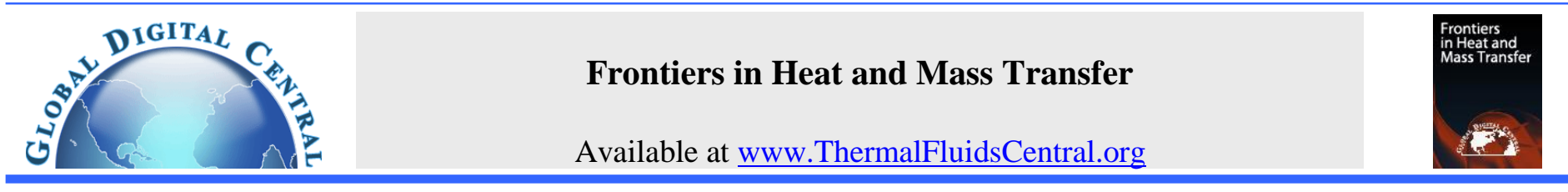

\title{
TREE-TYPE NANOPOROUS FILTERING MEMBRANE WITH COMPLEX PORES
}

\author{
Yongbin Zhang* \\ College of Mechanical Engineering, Changzhou University, Changzhou, Jiangsu, 21364, China
}

\begin{abstract}
A tree-type nanoporous filtering membrane with complex pores is proposed. The membrane consists of three kinds of concentric cylindrical pores across the membrane thickness i.e. the four branch pores for filtration, the one flow-collecting pore and the one flow resistance-reducing pore. The ratio of the radius of the flow resistance-reducing pore to that of the filtration pore is optimized for yielding the highest flux of the membrane. The dimensionless lowest flow resistance of the membrane in the optimum condition was typically calculated for different filtration pore radii and different passing liquid-pore wall interactions. The capability of the membrane for a liquid-liquid separation was also investigated. It was found that the proposed membrane has improved manufacturing properties without loss of the mechanical strength, the high flux and the liquid-particle or liquid-liquid separation capabilities.
\end{abstract}

Keywords: Membrane; Nanopore; Filtration; Flux; Flow resistance

\section{INTRODUCTION}

Nanoporous filtering membranes have been widely used in ultimate purification of water and seawater, drug delivery, virus filtration, DNA analysis, biological fuel cells and biological sensors et al. (Biffinger et al., 2007; Das et al., 2014; Desai et al., 2000; Escosura and Merkoci, 2011; Fissel et al., 2009; Gong et al., 2003; Gultepe et al., 2010; Han et al., 2013; Iqbal et al., 2007; Jackson and Hillmyer, 2010; Kim et al., 2006; Venkatesan et al., 2009; Yang et al., 2008). The challenging to such membranes is the flux and the mechanical strength of the membrane, both of which are limited because of the nanoscale filtration pore and the very small thickness of the membrane. For improving the performances of such membranes, novel nanoporous filtering membranes have been invented. Thin film composite membranes consist of a selective nanoporous layer and the supporting microporous layer and were found to have high fluxes (Cadotte et al.,1980; Tiraferri et al., 2011; Yip et al., 2010). A nanoporous membrane made of a mono layer graphene was found to have a high flux in water desalination (Surwade et al., 2015). Conical-shaped nanoporous membranes were shown to be advantageous over cylindrical-shaped nanoporous membranes because of higher fluxes (Li et al., 2004). Yang et al. (2006) proposed a mixed membrane consisting of the top layer with cylindrical nanopores and the bottom layer with micropores. They found a good performance of this membrane.

In the earlier study (Zhang, 2018a), an optimized cylindrical-shaped nanoporous filtering membrane was proposed. This membrane consists of two kinds of concentric cylindrical pores across the membrane thickness i.e. the filtration pore and the flow resistance-reducing pore; The ratio of the radius of the flow resistance-reducing pore to that of the filtration pore can be optimized for yielding the highest flux of the membrane. This membrane was also found to be capable of filtering one liquid out of other liquids (Zhang, 2017a). Later, a tree-type cylindricalshaped nanoporous filtering membrane was proposed (Zhang, 2018b). This membrane has tree-structured pores across the membrane thickness which consist of four identical branch pores and one bigger trunk pore; The trunk pore is for collecting the flow out of its branch pores and for reducing the flow resistance and thus increasing the flux of the membrane because of its bigger radius. It was suggested that both the flux and the mechanical strength of this membrane are considerably improved (Zhang, 2018b).

The present paper proposes a tree-type nanoporous filtering membrane across the thickness of which are respectively manufactured three kinds of cylindrical pores i.e. the four branch pores, the one flowcollecting pore and the one trunk pore. The branch pore is for filtration, the flow-collecting pore is bigger and just for collecting the flow out of its branch pores, and the radius of the trunk pore is optimized for yielding the highest flux of the membrane. The analysis for this membrane is presented when neglecting the liquid-pore wall interfacial slippage. The optimal ratio of the radius of the trunk pore to that of the branch pore is calculated for different filtration pore radii and different passing liquidpore wall interactions. The dimensionless lowest flow resistance of this membrane in the optimum condition is calculated for a typical case. The capability of this membrane for a liquid-liquid separation is also investigated.

\section{STUDIED MEMBRANE}

Figs.1(a) and (b) show the studied cylindrical-shaped nanoporous filtering membrane, which has tree-structured pores across the membrane thickness. In each pore tree, there are four identical branch pores for filtration linked with one flow-collecting pore with a bigger radius, and the liquid flows through the flow resistance-reducing pore 
and then out of the membrane. The radius of the flow resistance-reducing pore can be optimized for yielding the lowest flow resistance then the highest flux of the membrane. There is an angle $\theta$ between the axis of each branch pore and that of the flow-collecting pore. The axial lengths of the branch pore, the flow-collecting pore and the flow resistancereducing pore are respectively $l_{1}, l_{2}$ and $l_{3}$. The radii of these pores are respectively $R_{b, 1}, R_{e q}$ and $R_{b, 2}$. The membrane thickness is $l$. The flow direction in each pore is also marked by the arrow in Fig.1(a).

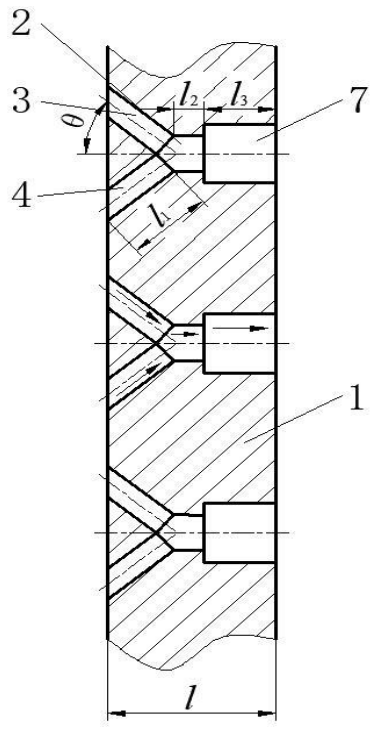

(a) Front view

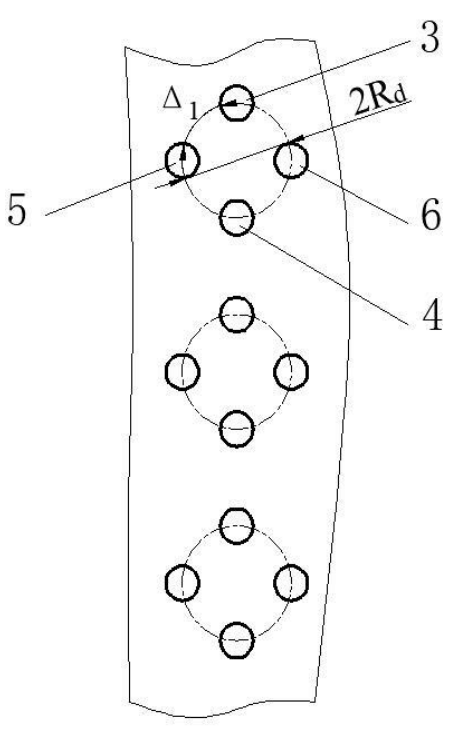

(b) Left-side view
1-Membrane substrate, 2-Flow-collecting pore, 3,4,5,6-Branch pores, 7-Flow resistance-reducing pore

Fig. 1 The studied tree-type cylindrical-shaped nanoporous filtering membrane with complex pores.

\section{ANALYSIS}

The flow within the nanopores in the membrane in Figs.1(a) and (b) is modeled by using the flow equation for a nanoscale flow (Zhang, 2016). For simplicity, the liquid-pore wall interfacial slippage is here neglected. According to the principle of the transportation in the nanotube tree given by the author (Zhang, 2017b), the four branch pores in Fig.1(a) are equivalent to the single straight cylindrical pore with the radius $R_{e q}$ and the axial length $l_{1}$. The radius $R_{e q}$ is solved from the following equation (Zhang, 2017b):

$\frac{4 C q\left(\bar{R}_{b, 1}\right)\left|S\left(\bar{R}_{b, 1}\right)\right| \bar{R}_{b, 1}^{4}}{C y\left(\bar{R}_{b, 1}\right)}-\frac{C q\left(\bar{R}_{e q}\right)\left|S\left(\bar{R}_{e q}\right)\right| \bar{R}_{e q}^{4}}{C y\left(\bar{R}_{e q}\right)}=0$

where $\bar{R}_{b, 1}=R_{b, 1} / R_{c r}, \bar{R}_{e q}=R_{e q} / R_{c r}, R_{b, 1}$ is the radius of the branch pore, $R_{c r}$ is the critical radius of the pore for the passing liquid to become continuum across the pore radius, $C y(\bar{R})=\eta_{b f}^{e f f}(\bar{R}) / \eta$, $C q(\bar{R})=\rho_{b f}^{\text {eff }}(\bar{R}) / \rho, \rho_{b f}^{\text {eff }}$ and $\eta_{b f}^{\text {eff }}$ are respectively the average density and the effective viscosity of the filtered liquid across the pore radius, $S$ is the parameter describing the non-continuum effect of the filtered liquid across the pore radius $(-1 \leq S<0)$, and $\rho$ and $\eta$ are respectively the bulk density and the bulk viscosity of the passing liquid at the environmental temperature and pressure.

Thus, the membrane in Fig.1(a) is equivalent to the membrane in Fig.2. The radii of the pores marked by the number " 2 " in these two figures are the same and equal to $R_{e q}$. The radii of the pores marked by the number "7" in these two figures both are equal to $R_{b, 2}$.

According to the study by Zhang (2018a), for yielding the lowest flow resistance of the membrane in Fig. 2, when $R_{b, 2} \geq 0.9 R_{c r}$, the optimum ratio of the radius $R_{b, 2}$ of the flow resistance-reducing pore to that $\left(R_{e q}\right)$ of the flow-collecting pore should be:

$\left(\frac{R_{b, 2}}{R_{e q}}\right)_{o p t}= \begin{cases}{\left[\frac{\left(1-\lambda_{0}\right) C q\left(\bar{R}_{e q}\right)\left|S\left(\bar{R}_{e q}\right)\right|}{\lambda_{0} C y\left(\bar{R}_{e q}\right)}\right]^{\frac{1}{4}}} & \text { for } R_{b, 2} \geq R_{e q} \\ 1 & \text { for } R_{b, 2}<R_{e q}\end{cases}$

where $\lambda_{0}=\left(l_{1}+l_{2}\right) /\left(l_{1}+l_{2}+l_{3}\right)$.

The corresponding resulting dimensionless lowest flow resistance of the membrane in Fig.2 is (Zhang, 2018a): $I_{f, \min }=\left(\bar{R}_{r} / \bar{R}_{e q}\right)^{2} F_{\text {min }}$, where $\bar{R}_{r}=R_{r} / R_{c r}, R_{r}$ is a constant reference radius, $I_{f, \min }=$ $\rho \chi A_{m} R_{r}^{2} i_{f, \min } /\left[4 \eta\left(l_{1}+l_{2}+l_{3}\right)\right], A_{m}$ is the area of the membrane surface, $\chi$ is the pore production rate of the membrane surface, $i_{f, \min }$ is the dimensional lowest flow resistance of the membrane $\left(i_{f, \min }=\right.$ $\Delta p / q_{m}, \Delta p$ is the pressure drop on the membrane, and $q_{m}$ is the mass flow rate through the membrane), and:

$F_{\text {min }}=\left\{\begin{array}{cl}2 \sqrt{\frac{\lambda_{0}\left(1-\lambda_{0}\right) C y\left(\bar{R}_{e q)}\right)}{C q\left(\bar{R}_{e q}\right) \mid S\left(\bar{R}_{e q}\right)}} & \text { for } R_{b, 2} \geq R_{e q} \\ \frac{C y\left(\bar{R}_{e q}\right)}{C q\left(\bar{R}_{e q}\right) \mid S\left(\bar{R}_{e q}\right)} & \text { for } R_{b, 2}<R_{e q}\end{array}\right.$

According to the analysis above, for yielding the lowest flow resistance of the membrane in Fig.1(a), the optimum ratio of $R_{b, 2}$ to $R_{b, 1}$ is: $\left(R_{b, 2} / R_{b, 1}\right)_{o p t}=\left(R_{e q} / R_{b, 1}\right)\left(R_{b, 2} / R_{e q}\right)_{o p t}$.

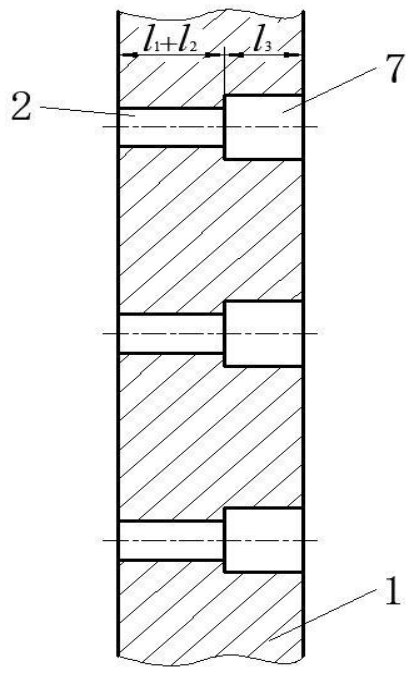

1-Membrane substrate, 2-Flow-collecting pore, 7-Flow resistancereducing pore

Fig. 2 The membrane equivalent to the membrane in Fig.1(a).

\section{CALCULATION}

The values of $\left(R_{b, 2} / R_{b, 1}\right)_{\text {opt }}$ and the corresponding lowest dimensionless flow resistance $I_{f, \min }$ of the membrane in Fig.1 were calculated. In the calculations, for whichever liquid-pore wall interaction, $C q(\bar{R})$ was generally expressed as (Zhang, 2017a,b and 2018b):

$C q(\bar{R})= \begin{cases}1 & , \text { for } \bar{R} \geq 1 \\ m_{0}+m_{1} \bar{R}+m_{2} \bar{R}^{2}+m_{3} \bar{R}^{3}, & \text { for } 0<\bar{R}<1\end{cases}$ 
where $\bar{R}$ is $\bar{R}_{b, 1}$ or $\bar{R}_{e q}$ (same in the following equations), $m_{0}, m_{1}$, $m_{2}$ and $m_{3}$ are respectively constants.

$C y(\bar{R})$ was generally expressed as (Zhang, 2017a,b and 2018b):

$\operatorname{Cy}(\bar{R})= \begin{cases}1 & , \text { for } \bar{R} \geq 1 \\ a_{0}+\frac{a_{1}}{\bar{R}}+\frac{a_{2}}{\bar{R}^{2}}, & \text { for } 0<\bar{R}<1\end{cases}$

where $a_{0}, a_{1}$ and $a_{2}$ are respectively constants.

$\mathrm{S}(\bar{R})$ was generally expressed as (Zhang, 2017a,b and 2018b):

$S(\bar{R})= \begin{cases}-1 & \text { for } \bar{R} \geq 1 \\ {\left[n_{0}+n_{1}\left(\bar{R}-n_{3}\right)^{n_{2}}\right]^{-1},} & \text { for } 0<\bar{R}<1\end{cases}$

where $n_{0}, n_{1}, n_{2}$ and $n_{3}$ are respectively constants.

For weak, medium-level and strong liquid-pore wall interactions, the values of $R_{c r}$ were respectively taken as $3.5 \mathrm{~nm}, 10 \mathrm{~nm}$ and $20 \mathrm{~nm}$ (Zhang, 2017a,b and 2018b). For different types of the passing liquidpore wall interaction, the values of the other parameters are respectively shown in Tables 1(a-c); These parameter values give different density and viscosity profiles across the pore radius and different non-continuum effects of the liquid in the nanopore respectively showing different liquid-pore wall interactions (Zhang, 2014).

Table 1(a) Liquid viscosity data for different liquid-pore wall interaction types (Zhang, 2017a, b and 2018b)

\begin{tabular}{|l|c|l|c|}
\hline \multicolumn{1}{|c|}{ Parameter } & $\mathrm{a}_{0}$ & $\mathrm{a}_{1}$ & $\mathrm{a}_{2}$ \\
\hline Interaction & & & \\
\hline Mtrong & 1.8335 & -1.4252 & 0.5917 \\
\hline Wedium & 1.0822 & -0.1758 & 0.0936 \\
\hline
\end{tabular}

Table 1(b) Liquid density data for different liquid-pore wall interaction types (Zhang, 2017a, b and 2018b)

\begin{tabular}{|l|c|c|c|c|}
\hline Interaction & \multicolumn{1}{|c|}{$\mathrm{m}_{0}$} & $\mathrm{~m}_{1}$ & $\mathrm{~m}_{2}$ & $\mathrm{~m}_{3}$ \\
\hline Strong & & & & \\
\hline Medium & 1.43 & -1.723 & 2.641 & -1.347 \\
\hline Weak & 1.30 & -1.065 & 1.336 & -0.571 \\
\hline
\end{tabular}

Table 1(c) Liquid non-continuum property data for different liquidpore wall interaction types (Zhang, 2017a, b and 2018b)

\begin{tabular}{|l|l|l|l|l|}
\hline Interaction & \multicolumn{1}{|c|}{$\mathrm{n}_{0}$} & $\mathrm{n}_{1}$ & $\mathrm{n}_{2}$ & $\mathrm{n}_{3}$ \\
\hline Strong & & & & \\
\hline Medium & 0.4 & -1.374 & -0.534 & 0.035 \\
\hline Weak & -0.649 & -0.343 & -0.665 & 0.035 \\
\hline
\end{tabular}

\section{RESULTS AND DISCUSSION}

Figures 3(a), (b) and (c) respectively show the values of $\left(R_{b, 2} / R_{b, 1}\right)_{\text {opt }}$ for different $R_{b, 1}$ and different liquid-pore wall interactions when $\lambda_{0}=$ $1 \times 10^{-4}, \lambda_{0}=1 \times 10^{-3}$ and $\lambda_{0}=1 \times 10^{-2}$. When $R_{b, 1}$ is on the $1 \mathrm{~nm}$ scale, the liquid-pore wall interaction has a significant influence on the value of $\left(R_{b, 2} / R_{b, 1}\right)_{\text {opt }}$. However, when $R_{b, 1}$ is so large that the flow inside the branch pore is nearly a continuum flow, the influence of the liquid-pore wall interaction on the value of $\left(R_{b, 2} / R_{b, 1}\right)_{\text {opt }}$ is negligible and the value of $\left(R_{b, 2} / R_{b, 1}\right)_{o p t}$ is independent on $R_{b, 1}$ but strongly dependent on $\lambda_{0}$. For a given $R_{b, 1}$ and a given liquid-pore wall interaction, the increase of $\lambda_{0}$ significantly reduces the value of $\left(R_{b, 2} / R_{b, 1}\right)_{\text {opt }}$. Figures 3(a),(b) and (c) show that for yielding the lowest flow resistance i.e. the highest flux of the membrane under a given pressure drop $\Delta p$, the radius $R_{b, 2}$ of the flow resistance-reducing pore should be several times or even more than ten times the radius $R_{b, 1}$ of the branch pore. This shows the evident benefits of the flow resistancereducing pore in improving the flux of the present membrane.

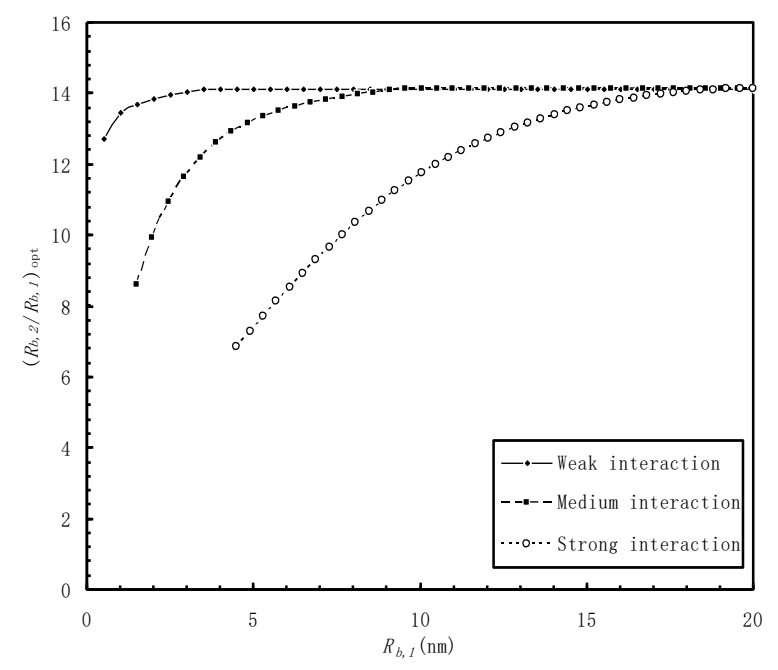

(a) $\lambda_{0}=1 \times 10^{-4}$

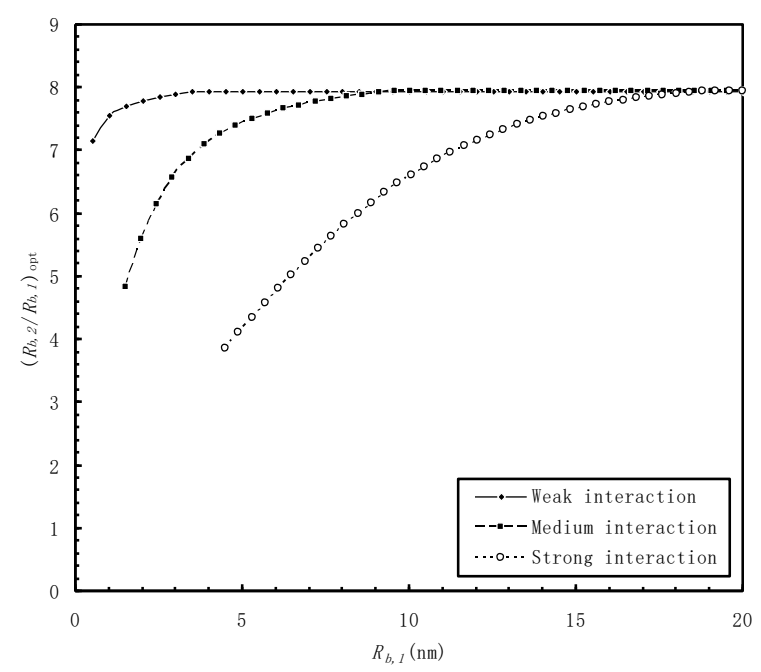

(b) $\lambda_{0}=1 \times 10^{-3}$

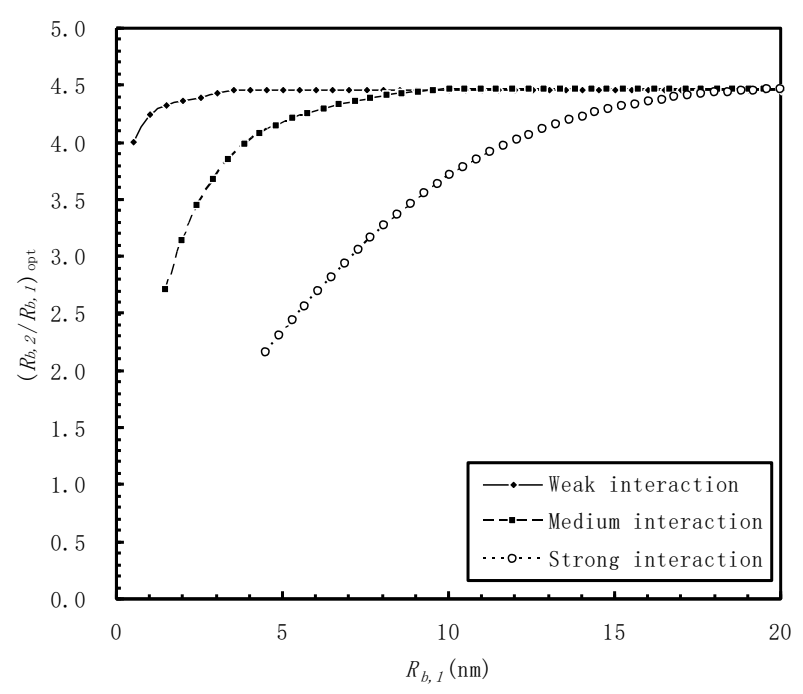

(c) $\lambda_{0}=1 \times 10^{-2}$

Fig. 3 Values of $\left(R_{b, 2} / R_{b, 1}\right)_{\text {opt }}$.

As an example, Fig.4 shows the values of the dimensionless lowest flow resistance $I_{f, \min }$ of the studied membrane for different $R_{b, 1}$ and different liquid-pore wall interactions when $\lambda_{0}=1 \times 10^{-3}$ and $R_{r}=$ 
$10 \mathrm{~nm}$. It is shown that in the optimum condition the flow resistance of the studied membrane is significantly reduced with the increase of $R_{b, 1}$ especially when $R_{b, 1}$ is on the $1 \mathrm{~nm}$ scale; For $R_{b, 1}$ on the $1 \mathrm{~nm}$ scale, the liquid-pore wall interaction has a significant influence on the flow resistance of the membrane, and a weak liquid-pore wall interaction results in a considerably lower flow resistance of the membrane and is thus significantly beneficial for the flux of the membrane; However, for $R_{b, 1}$ about over $15 \mathrm{~nm}$, the liquid-pore wall interaction has a negligible influence on the flow resistance and thus the flux of the membrane.

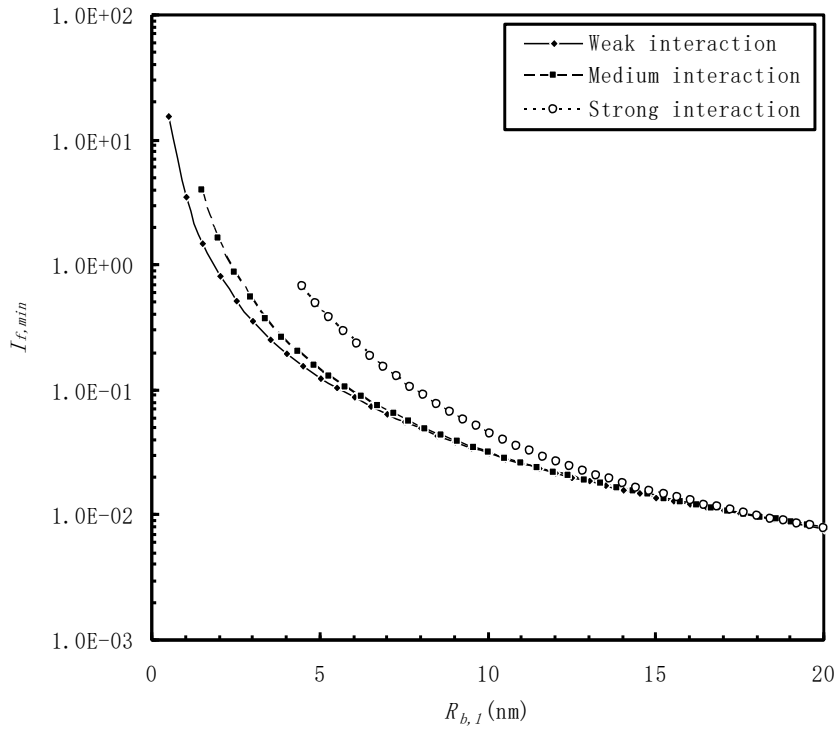

Fig. 4 Values of the dimensionless lowest flow resistance $I_{f, \min }$ of the studied membrane when $\lambda_{0}=1 \times 10^{-3}$ and $R_{r}=10 \mathrm{~nm}$.

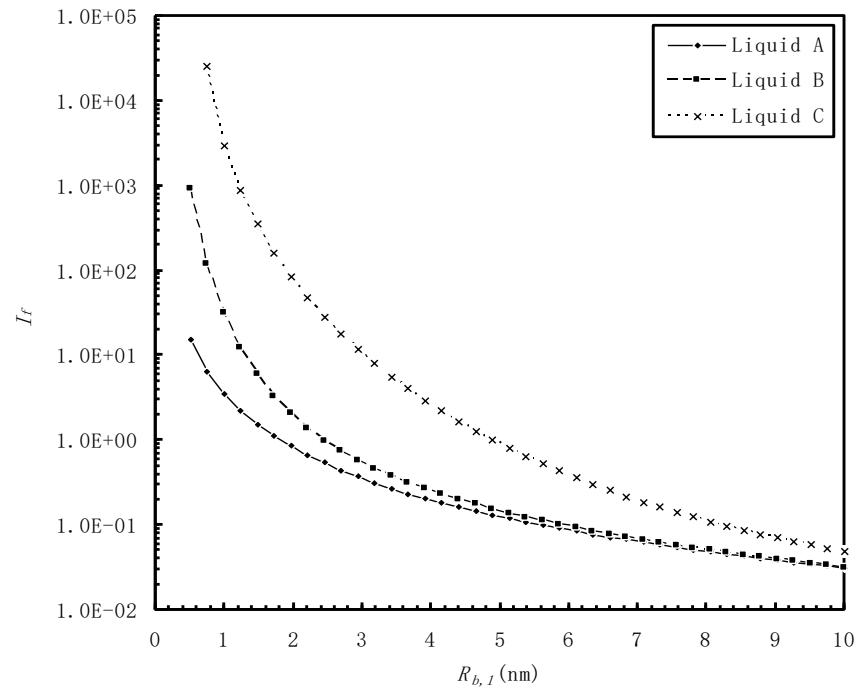

Fig. 5 Values of the dimensionless flow resistance $I_{f}$ of the studied membrane respectively for Liquid $\mathrm{A}$, Liquid $\mathrm{B}$ and Liquid $\mathrm{C}$ when $\lambda_{0}=1 \times 10^{-3}, R_{r}=10 \mathrm{~nm}$, and the ratio of $R_{b, 2}$ to $R_{b, 1}$ is optimum determined according to Liquid A.

Figure 5 shows the values of the dimensionless flow resistance $I_{f}$ of the studied membrane respectively for Liquid A, Liquid B and Liquid $\mathrm{C}$ when $\lambda_{0}=1 \times 10^{-3}, R_{r}=10 \mathrm{~nm}$, and the ratio of $R_{b, 2}$ to $R_{b, 1}$ is optimum determined according to Liquid A (Zhang, 2018a). Here, Liquid A has a weak interaction with the pore wall, Liquid B has a medium-level interaction with the pore wall, and Liquid $\mathrm{C}$ has a strong interaction with the pore wall. It is shown that when $R_{b, 1}$ is lower than
$2.5 \mathrm{~nm}$, the flow resistance of the membrane for Liquid $\mathrm{C}$ is more than 50 times or even more than 3000 times that for Liquid A. This provides an indication that the present membrane can be used for a liquid-liquid separation provided that the mixed liquids have greatly different interactions with the pore wall. In this application, the liquid which has a weak interaction with the pore wall can freely pass through the membrane, while the liquid which has a strong interaction with the pore wall can hardly pass through the membrane.

\section{CONCLUSIONS}

The present paper analytically investigates the performance of a treetype cylindrical-shaped nanoporous filtering membrane with complex pores based on the developed equation for a nanoscale fluid flow. The membrane has tree-structured pores across the membrane thickness. In each pore tree, there are four identical branch pores for filtration, one flow-collecting pore and one flow resistance-reducing pore. The radius of the flow-collecting pore is calculated from the derived equation according to the requirement of this pore for just collecting the flow out of its branch pores. The radius of the flow resistance-reducing pore is optimized for yielding the lowest flow resistance of the membrane.

The optimum ratios of the radius of the flow resistance-reducing pore to that of the branch pore were calculated for practical operational parameter values and different passing liquid-pore wall interactions. The corresponding dimensionless lowest flow resistance of the membrane was also typically calculated. It was found that the flow resistancereducing pore is very beneficial for reducing the flow resistance and thus for improving the flux of the membrane, and its radius is normally much greater than the radius of the branch pore; In the optimum condition, the radius of the branch pore has a great influence on the flow resistance and thus on the flux of the membrane; When the radius of the branch pore is on the $1 \mathrm{~nm}$ scale, the passing liquid-pore wall interaction has a significant influence on the flow resistance of the membrane, otherwise this influence may be weak or even negligible.

The studied membrane was found to be capable of filtering one liquid out of other liquids provided that the mixed liquids have greatly different interactions with the pore wall. For a liquid-liquid separation, the optimum radius of the flow resistance-reducing pore should be determined according to the liquid which has a weak interaction with the pore wall. In this condition, when the radius of the branch pore is no more than $2.5 \mathrm{~nm}$, the liquid which has a weak interaction with the pore wall can freely pass through the membrane, while the other liquids can hardly pass through the membrane, because of the largely different flow resistances of the membrane respectively for these liquids.

Due to the significantly higher membrane flux in the same operating condition, the tree-type membrane in this study (with four branch pores in each pore tree) is advantageous over the concentric cylindrical-shaped nanoporous filtration membrane proposed earlier (Zhang, 2018a), which actually only has one branch pore in each pore tree.

\section{REFERENCES}

Biffinger, J. C., Ray, R., Little, B., Ringeisen, B. R., 2007, “Diversifying Biological Fuel Cell Designs by Use of Nanoporous Filters,"Environmental Science \& Technology, 41, 1444-1449. https://doi.org/10.1021/es061634u

Cadotte, J. E., Petersen, R. J., Larson, R. E., Erickson, E. E., 1980, “A new thin film composite seawater reverse osmosis membrane," Desalination, 32, 25-31. https://doi.org/10.1016/S0011-9164(00)86003-8

Das, R., Eaqub Ali, Md, Abd Hamid, S. B., Ramakrishna, S., and Chowdhury, Z. Z., 2014, "Carbon Nanotube Membranes for Water Purification: A Bright Future in Water Desalination," Desalination, 336, 97-109.

https://doi.org/10.1016/j.desal.2013.12.026 
Desai, T. A., Hansford, D. J., Leoni, L., Essenpreis, M., Ferrari, M., 2000 "Nanoporous Anti-Fouling Silicon Membranes for Biosensor Applications," Biosensors and Bioelectronics, 15, 453-462. https://doi.org/10.1016/S0956-5663(00)00088-9

Escosura, A., Merkoci, A., 2011, “A Nanochannel/Nanoparticle-Based Filtering and Sensing Platform for Direct Detection of a Cancer Biomarker in Blood," Small, 7, 675-682. https://doi.org/10.1002/smll.201002349

Fissel, W. H., Dubnisheva, A., Eldridge, A. N., Fleischman, A. J., Zydney, A. L., and Roy, S., 2009, "High-Performance Silicon Nanopore Hemofiltration Membranes," Journal of Membrane Science, 326, 58-63. https://doi.org/10.1016/j.memsci.2008.09.039

Gong, D., Yadavalli, V., Paulose, M., Pishko, M., Grimes, C. A., 2003, "Controlled Molecular Release Using Nanoporous Alumina Capsules," Biomedical Microdevices, 5, 75-80. https://doi.org/10.1023/A:1024471618380

Gultepe, E., Nagesha, D., Sridhar, S., Amiji, M.,2010, "Nanoporous inorganic membranes or coatings for sustained drug delivery in implantable devices," Advanced Drug Delivery Reviews, 62, 305-315. https://doi.org/10.1016/j.addr.2009.11.003

Han, Y., Xu, Z., and Gao, C., 2013, "Ultrathin Graphene Nanofiltration Membrane for Water Purification," Advanced Functional Materials, 23, 3693-3700

https://doi.org/10.1002/adfm.201202601

Iqbal, S. M., Akin, D., Bashir, R., 2007, "Solid-State Nanopore Channels with DNA Selectivity," Nature Nanotechnology, 2, 243-248. http://doi:10.1038/nnano.2007.78

Jackson, E. A. and Hillmyer, M. A., 2010, "Nanoporous membranes derived from block copolymers: From drug delivery to water filtration," ACS Nano, 4, 3548-3553. https://doi.org/10.1021/nn1014006

Kim, M. J., Wanunu, M., Bell, D. C., and Meller, A., 2006, "Rapid Fabrication of Uniformly Sized Nanopores and Nanopore Arrays for Parallel DNA Analysis," Advanced Materials, 18, 3149-3153. https://doi.org/10.1002/adma.200601191

Li, N., Yu, S., Harrell, C., and Martin, C. R., 2004, "Conical Nanopore Membranes: Preparation and Transport Properties,"Analytical Chemistry, 76, 2025-2030.

https://doi.org/10.1021/ac035402e

Surwade, S. P., Smirnov, S. N., Vlassiouk, I. V., Unocic, R. R., Veith, G. M., Dai, S., and Mahurin, S. M., 2015, "Water Desalination Using Nanoporous Single-Layer Grapheme," Nature Nanotechnology, 10, 459464.

https://doi.org/10.1038/nnano.2015.37
Tiraferri, A., Yip, N. Y., Phillip, W. A., Schiffman, J. D., Elimelech, M., 2011, "Relating Performance of Thin-Film Composite Forward Osmosis Membranes to Support Layer Formation and Structure," Journal of Membrane Science, 367, 340-352.

https://doi.org/10.1016/j.memsci.2010.11.014

Venkatesan, B. M., Dorvel, B., Yemenicioglu, S., Watkins, N., Petrov, I., and Bshir, R., 2009, "Highly Sensitive, Mechanically Stable Nanopore Sensors for DNA Analysis," Advanced Materials, 21, 2771-2776. https://doi.org/10.1002/adma.200803786

Yang, S. Y., Ryu, I., Kim, H. Y., Kim, J. K., Jang, S. K., and Russell, T. P., 2006, "Nanoporous Membranes with Ultrahigh Selectivity and Flux for the Filtration of Viruses," Advanced Materials, 18, 709-712. https://doi.org/10.1002/adma.200501500

Yang, S. Y., Park, J., Yoon, J., Ree, M., Jang, S. K., Kim, J. K., 2008, "Virus Filtration Membranes Prepared from Nanoporous Block Copolymers with Good Dimensional Stability under High Pressures and Excellent Solvent Resistance,"Advanced Functional Materials, 18, 1371-1377.

https://doi.org/10.1002/adfm.200700832

Zhang, Y. B., 2014, "Lubrication Analysis for a Line Contact Covering From Boundary Lubrication to Hydrodynamic Lubrication: Part IMicro Contact Results," Journal of Computational and Theoretical Nanoscience., 11, 62-70.

https://doi.org/10.1166/jctn.2014.3318

Yip, N. Y., Tiraferri, A., Phillip, W. A., Schiffman, J. D., Elimelech, M., 2010, "High Performance Thin-Film Composite Forward Osmosis Membrane," Environment Science and Technology, 44, 3812-3818. https://doi: 10.1021/es1002555

Zhang, Y. B., 2017a, "Influence of Pore Wall Surface Property on Flux of Cylindrical-Shaped Nanoporous Filtering Membrane," Frontiers in Heat and Mass Transfer, 9, 26.

https://doi.org/10.5098/hmt.9.26

Zhang, Y. B., 2017b, "Transport in Nanotube Tree," International Journal of Heat and Mass Transfer, 114, 536-540. https://doi.org/10.1016/j.ijheatmasstransfer.2017.06.105

Zhang, Y. B., 2018a, "Optimum Design for Cylindrical-Shaped Nanoporous Filtration Membrane," International Communications in Heat and Mass Transfer, 96, 130-138. https://doi.org/10.1016/j.icheatmasstransfer.2018.06.003

Zhang, Y. B., 2018b, "A Tree-Type Cylindrical-Shaped Nanoporous Filtering Membrane," Frontiers in Heat and Mass Transfer, 10, 16. https://doi:10.5098/hmt.10.16 